\title{
Imprevisibilidade familiar e perspectiva temporal de futuro: Retro-FUS versão portuguesa
}

\section{Family unpredictability and future time perspective: Portuguese Retro-FUS}

\author{
Teresa Sousa Machado, Pedro Tiago Pereira, José Tomás da Silva, José Pacheco Miguel \\ Faculdade de Psicologia e de Ciências da Educação, Universidade de Coimbra
}

\begin{abstract}
Resumo
A imprevisibilidade familiar, ou inconsistência dos cuidados e regulação familiar, associa-se a problemas de comportamento e ao desenvolvimento inadaptado dos filhos. A imprevisibilidade pode dever-se ao risco ambiental (pobreza, emigração, desemprego), ou stressores individuais e familiares (e.g., divórcio, alcoolismo, doença mental). O comprometimento das rotinas que garantem segurança e estabilidade, subjaz à imprevisibilidade, dificultando a criação de expectativas futuras. Os dados apresentados resultam da primeira versão para português da Retro-FUS (com 261 universitários, entre 18 e 29 anos), e de análises de correlação entre imprevisibilidade retrospectiva e perspectiva de futuro. Correlações negativas entre os constructos reforçam o modelo teórico subjacente.

Palavras chave: Retro-FUS, Imprevisibilidade familiar, perspectiva temporal de futuro
\end{abstract}

\begin{abstract}
Family unpredictability, as inconsistency of parental behavior in meeting children's needs and family regulation, has been associated with problem behavior, and psychopathology development. Unpredictability can occur in environmental risk conditions (poverty, emigration, unemployment), or be associated with parental stressors (divorce, alcoholism, mental diseases). The common point of these stressors is that they compromise familiar routines that enable security, and predictability. We present data from the first Portuguese translation and adaptation of Retro-FUS (261 College young adults, aged from 18 to 29). Negative correlations between retrospective family unpredictability and future time perspective corroborate the theoretical model.

Keywords: Retro-FUS, family unpredictability, future time perspective
\end{abstract}

A imprevisibilidade familiar, ao impedir a manutenção de rotinas e o cumprimento de expectativas relativas às dinâmicas familiares e relacionais, tem sido associada a perturbações no desenvolvimento das crianças. A literatura mostra que essa imprevisibilidade induz stresse e desgaste pessoal, dificultando o planeamento e realização de acções que permitem pre-estruturar o futuro. A situação social e económica contemporânea, pautada por instabilidade no emprego e decréscimo dos vencimentos (nomeadamente em Portugal), contribui para o aumento do sentimento de imprevisibilidade nas famílias, reforçando a tese sobre a existência de uma relação entre economia e estrutura e qualidade da vida familiar (Larson, Wilson, \& Beley, 1994; Voydanoff, 1990, 2005). Mais ainda, confirmando a relação entre a satisfação no trabalho e a satisfação marital e familiar (Voydanoff, 2005).

Estudos sobre a temática têm validado a ideia de que a imprevisibilidade familiar - enquanto ausência de padrões estáveis de conduta e rotinas que estruturam o dia-a-dia das crianças e família - se associa a mais disfunções familiares e mais comportamentos de risco dos filhos; levando, por exemplo, os sujeitos a não se protegerem de doenças, de gravidezes indesejadas, e a envolverem-se mais facilmente em comportamentos antissociais (Hill, Ross, \& Low, 1997; Hill, Jenkins, \& Farmer, 2008). Ou seja, a imprevisibilidade familiar propicia, ao longo do desenvolvimento dos filhos, uma cascada de comportamentos negativos, com efeitos a longo prazo (Alarcão \& Gaspar, 2007; Jenkins, \& Farmer, 2008; Hill, Young, \& Nord, 1994; Howat-Rodrigues, Andrade, \& Tokumaru, 2012; Ross \& Hill, 2000, 2002).

Esta imprevisibilidade na família diz respeito à percepção de que o ambiente não é controlável, o que leva à construção de representações de "falta de controlo para regular e organizar acontecimentos e disponibilidade sazonal de recursos" (Howat-Rodrigues, Andrade, \& Tokumaru, 2012, p.212). É a inconsistência do comportamento parental no cumprimento do seu papel que gera esta imprevisibilidade.

De acordo com Ross e McDuffy (2008), o conceito de imprevisibilidade familiar deriva simultaneamente da teoria da vinculação de Bowlby e da teoria do desânimo aprendido, de Seligman (1975). A experiência de Seligman e Maier (1967), mostrou que quando uma resposta a um estímulo é aleatória (ou seja, não contingente a algo que um animal fez), este desiste de tentar, pois aprendeu que a relação entre comportamento e resultado é independente. Ou seja, quando os indivíduos veem as suas acções como irrelevantes para os resultados subsequentes, desenvolvem o que se designa por "desânimo aprendido", traduzido na desistência de encetar novos esforços (Trad, 1986). Este efeito tem sido reconfirmado, (desde os trabalhos de Seligman em 1965), em estudos experimentais com animais (Hunziker, 2005; Hunziker, \& dos Santos, 2007; Oliveira, \& Hunziker, 2014; Seligman \& Maier, 1967), e com humanos (Kilday, 2013; Nenty \& Edna, 2009; Sorrenti, Filipello, Costa, \& Buzzai, 2014). O efeito pode ser global, ou específico a determinados comportamentos ou temáticas (e.g., desânimo e desistência relativa a esforços em desempenhos académicos específicos). $\mathrm{O}$ sujeito sente que não tem controlo sobre o seu destino, e 
desiste de ensaiar novas respostas, conformando-se com a situação, o que leva à inibição de novas aprendizagens (Kilday, 2013; Nenty \& Edna, 2009). Ross, Hill, e McDuffy, entre outros, têm vindo a mostrar que a imprevisibilidade familiar leva a resultados semelhantes: i.e., à ideia de que planear estratégicas com vista a um dado fim tem grandes hipóteses de falhar, pelo que não vale a pena encetá-las. A hipótese que aqui estudamos, com jovens adultos, é de que a recordação de imprevisibilidade familiar em jovens adultos (i.e., relativa à sua infância/adolescência) influencia a estruturação da perspectiva temporal de futuro, debilitando a ideia de que faz sentido, e é possível, preparar o futuro. Nas famílias pautadas por imprevisibilidade, o facto dos comportamentos dos cuidadores não serem contingentes aos comportamentos dos filhos, leva a que os feedbacks desses cuidadores não sejam efectivos; impedindo (ou dificultando) as crianças de construírem padrões de resposta, bem como minando a crença de que o futuro pode ser planeado (LoLordo, \& Overmier, 2011). Ora, a capacidade para planear o futuro, e correlativamente, para adiar determinadas realizações (e.g., ter um filho, terminar uma graduação) é essencial para a própria sobrevivência (real e simbólica) do homem (Bandura, 2001).

A consciencialização de uma dimensão temporal da vida implica a admissão de um passado, a noção do presente, e a existência de um futuro que se constrói (ou idealiza), estando estas dimensões relacionadas entre si, e encontrando-se aqui a força motivacional que impele o sujeito à acção (i.e., construção de um futuro planeado). A perspectiva temporal de futuro é, neste sentido, uma característica cognitiva-motivacional da personalidade, que permite estruturar objectivos e elaborar planos aos quais o sujeito atribui relevância (Husman, \& Shell, 2008; Lens, Paixão, Herrera, \& Grobler, 2012; Paixão, 2004). Para a construção dessa perspetiva temporal é necessário ter tido um passado pautado por uma certa consistência e previsibilidade, particularmente nos primeiros anos de vida, quando se constroem os primeiros modelos internos do self e das relações (Bretherton, \& Munholland, 2008; Machado 2007). Embora tais modelos permaneçam abertos à mudança, eles tendem a tornar-se mais "automáticos" ao longo do desenvolvimento.

Os efeitos nefastos da imprevisibilidade familiar têm sido confirmados em situações diversas que induzem quebras aleatórias nas rotinas familiares, como ter pais alcoólicos (Nodar, 2012, Ross, \& Hill, 2004), doenças crónicas do cuidador (Pakenham, \& Bursnall, 2006), depressão e divórcio dos pais (Ross, \& Wynne, 2010), carências económicas (Howat-Rodrigues, \& Tokumaru, 2014), insegurança no emprego (Larson, Wilson, \& Beley, 1994), entre outras. Não é a situação em si mesma a causa dessa imprevisibilidade, mas as implicações da situação; por exemplo, no caso do divórcio dos pais, os estudos sugerem que os efeitos nefastos manifestam-se usualmente antes do próprio divórcio, sugerindo que é a fraca qualidade da vida familiar que os induz, e não propriamente o divórcio em si mesmo (Ross \& McDuff, 2008; Ross, \& Wynne, 2010) - pelo que a intervenção para compensar a imprevisibilidade, poderá dirigir-se precisamente à atenuação desses efeitos (e.g., tentar preservar rotinas das crianças, como manter os mesmos amigos, a escola, e apoios ao cuidador). Ou, em situações de risco ambiental e/ou biológico, recorrer a apoios externos aos cuidadores das crianças (e.g., recorrer a programas de intervenção precoce na infância) (Machado, \& Correia, 2013).

Por seu turno, a teoria da vinculação mostra como, pelo contrário, as rotinas, (i.e., a previsibilidade), nos cuidados primários possibilitam a construção de modelos coesos e adaptativos do self e das relações. Ou seja, leva à convicção do efeito do comportamento do sujeito nos outros, oferecendo segurança ao bebé, que pode assim, progressivamente, antecipar respostas dos cuidadores face aos seus próprios comportamentos - o que cria a previsibilidade relacional que confere resistência (Bowlby, 1988; Bretherton, \& Munholland, 2008; Machado, 2004; 2009). A mesma teoria mostra como o padrão de vinculação ansioso-ambivalente (e num grau mais extremo, o padrão desorganizado), são, entre outros factores, influenciados pela imprevisibilidade das respostas dos cuidadores; compreendendo-se que se encontrem relações significativas entre a vinculação segura (aos pais e pares), a previsibilidade familiar e a perspectiva temporal de futuro (O’Dwyer, 2000). Laghi e colaboradores mostram que os adolescentes com padrões mais seguros aos pais apresentaram, na escala de perspectiva temporal (de Zimbardo), scores mais elevados nas dimensões "passado positivo", "presente hedonista", e "futuro" (Laghi, D’Alessio, Pallini, \& Baiocco, 2009). E, em geral, a orientação para o futuro tem sido associada a diversas consequências positivas em indivíduos das sociedades ocidentais (Zimbardo, \& Boyd, 1999). Compreende-se, desta forma, que os conceitos teóricos de imprevisibilidade familiar, desânimo aprendido, perspectiva temporal de futuro e vinculação se possam conjugar na explicitação dos efeitos desenvolvimentais da (im)previsibilidade vivenciada (ao longo da infância) na família.

Analisam-se, neste trabalho, as relações entre a imprevisibilidade familiar retrospectiva (i.e. recordações relativas à previsibilidade/imprevisibilidade das relações e dinâmicas familiares) e a perspectiva de futuro em jovens adultos universitários. Os dados da escala de imprevisibilidade retrospectiva são relativos à primeira tradução e adaptação para português da Retro-FUS (Ross, \& McDuff, 2008).

\section{Método}

\section{Participantes}

A amostra foi constituída por 261 jovens adultos, estudantes de instituições do Ensino Superior da Universidade de Coimbra.

As idades variam entre os 18 e 34 anos $(\mathrm{M}=21.3$; $\mathrm{DP}=$ $3.12)$; sendo $\mathrm{N}=131(50 \%)$ raparigas e $50 \%$ rapazes. 159 dos jovens $(60.7 \%)$ encontravam-se no $3^{\circ}$ ou $4^{\circ}$ ano de um curso superior, e $103(39.3 \%)$ no $1^{\circ}$ ou $2^{\circ}$ ano; 184 $(70.1 \%)$ frequentam cursos nas engenharias, e 78 $(29.9 \%)$ cursos nas ciências sociais e humanas (psicologia, ciências da educação, serviço social, e direito). 
A maioria pertence ao nível socioeconómico médio $(\mathrm{N}=109 ; 41.8 \%)$, repartindo-se os restantes pelos níveis baixo $(\mathrm{N}=99 ; 37.9 \%)$, e elevado $(\mathrm{N}=53 ; 20.2 \%)$.

\section{Instrumentos}

Escala Retrospectiva de Imprevisibilidade Familiar, versão portuguesa da Retro-Family Unpredictability Scale (Ross, \& McDuff, 2008), composta por 24 itens, e apresentando um valor de alpha .88, para escala global (depois de excluídos 4 itens da versão original), avalia a recordação da imprevisibilidade familiar vivenciadas ao longo da infância/adolescência do sujeito (e.g., "Eu podia contar com a minha mãe para me levar onde eu precisasse"; "Quando me sentia magoado (sentimentos) ia ter com o meu pai para me confortar"). Foi construída a partir da anterior Escala de Imprevisibilidade Familiar de Ross e Hill (2000), comportando as mesmas cinco dimensões relativas a dinâmicas familiares. É um instrumento de auto-relato no qual o sujeito avalia a "(im)previsibilidade" das rotinas habituais nas dinâmicas familiares mais comuns: i.e., disciplina, afecto-Pai, afecto-Mãe, refeições, finanças (na presente versão a dimensão disciplina surge apenas num factor, ao invés do que acontece na versão original na qual se subdivide em disciplina-Mãe e disciplina-Pai). A dimensão disciplina apresenta um alpha de .90, afecto paterno $\alpha=$ .83 , afecto materno $\alpha=.76$, refeições $\alpha=.67$, e finanças $\alpha$ $=.55$. Cotada numa escala de Likert de 1 a 5 pontos, maior pontuação traduz uma maior recordação de imprevisibilidade familiar relativa ao passado desenvolvimental do sujeito.

A versão portuguesa da Future Perspective Scale (Husman \& Shell, 2007), foi utilizada para avaliar a perspectiva do sujeito relativamente ao seu futuro. Constituída por 27 itens, engloba quatro áreas, ou dimensões, que dizem respeito à forma como o sujeito encara o seu futuro: valência (e.g., "Tendo hipótese de escolha, é preferível conseguirmos algo que desejamos no futuro, a ter algo que queremos agora"); extensão (e.g., "Janeiro, parece-me muito próximo"); conectividade ("Não penso muito no futuro"); rapidez ("Para mim é difícil conseguir coisas sem ter um prazo final"). É cotada numa escala Likert de 1 a 5 pontos; maior cotação traduz maior perspectiva temporal de futuro.

\section{Procedimento}

Após a autorização, por via electrónica, dos autores da Retro-FUS, procedeu-se à tradução e estudos de adaptação para português. A recolha da amostra foi realizada em diferentes Faculdades da Universidade de Coimbra, sempre em sala de aula, com presença do investigador, após autorização dos Professores responsáveis. $\mathrm{O}$ preenchimento dos dois instrumentos demorou entre 10 a 15 minutos.

\section{Resultados}

Após as primeiras análises relativas ao estudo da dimensionalidade e fiabilidade da versão portuguesa da Retro-FUS (apresentadas num outro trabalho), avaliaram-se as correlações entre as dimensões da Retro-FUS, na presente amostra. Os valores mostram que todas as dimensões se correlacionam entre si, sugerindo avaliar o mesmo constructo (Tabela 1.).

Tabela 1. Correlações entre dimensões da Retro-FUS

\begin{tabular}{|c|c|c|c|c|c|}
\hline & (1) & (2) & (3) & (4) & (5) \\
\hline Disciplina (1) & & $.34 * *$ & $.32 * *$ & $.28 * *$ & $.18^{* *}$ \\
\hline Afeto Pai (2) & & & $.57 * *$ & $.13^{* *}$ & $.19^{* *}$ \\
\hline Afeto Mãe (3) & & & & $.14^{* *}$ & $.22 * *$ \\
\hline Finanças (4) & & & & & $.18^{* *}$ \\
\hline Refeições (5) & & & & & \\
\hline
\end{tabular}

Em seguida apresentam-se as correlações (elevadas e significativas) entre cada dimensão da imprevisibilidade familiar recordada, e o valor total da escala, i.e., o score relativo à imprevisibilidade retrospectiva (global), e relativa às dinâmicas familiares avaliadas (Tabela 2.).

Tabela 2. Correlações entre dimensões Retro-FUS e Retro-FUS total

\begin{tabular}{lc}
\hline & Retro-FUS(total) \\
\hline Disciplina & $.79 * *$ \\
Afeto paternal & $.75 * *$ \\
Afeto maternal & $.71 * *$ \\
Finanças & $.40 * *$ \\
Refeições & $.39 * *$ \\
\hline $\mathrm{p} \leq .01$ &
\end{tabular}

Procedeu-se ao estudo das relações entre a recordação da imprevisibilidade familiar vivenciada ao longo da infância/adolescência dos sujeitos (nas suas dimensões e score global), e a perspectiva temporal de futuro dos jovens (dimensões e score global) (Tabela 3.).

Tabela 3. Correlações entre Retro-FUS e FTPS

\begin{tabular}{lccccc}
\hline & Valência & Conect. & Rapidez & Extens. & FTPS \\
\hline Disciplina & $.15 *$ & -.10 & $-.30 * *$ & -.08 & -.10 \\
Afeto Pai & .05 & -.04 & $-.20 * *$ & .01 & -.05 \\
Afeto Mãe & .04 & -.12 & $-.17 * *$ & .06 & $-.13 *$ \\
Finanças & .03 & $-.26 * *$ & .04 & .03 & $-.17 * *$ \\
Refeições & .10 & $-.17 * *$ & $-.16 *$ & -.08 & $-.14 *$ \\
Retro-FUS & $.12 *$ & $-.16^{* *}$ & $-.29 * *$ & -.07 & $-.15^{*}$ \\
\hline$* \mathrm{p} \leq .05 ; * * \mathrm{p} \leq .01$ & & & &
\end{tabular}

Em termos globais - isto é, considerando o score total da escala Retro-FUS e score total da FTPS podemos confirmar que maior imprevisibilidade familiar recordada (i.e., o score global da Retro-FUS) se correlaciona negativa e significativamente com a perspectiva temporal de futuro $(\mathrm{r}=-.15, p \leq .05)$, embora as correlações sejam de magnitude baixa (Cohen, 1988). Particularizando, as dimensões da Retro-FUS finanças, disciplina, refeições, afeto paterno e afeto materno, todas elas se correlacionam negativamente com dimensões relativas à perspectiva temporal de futuro; isto é, encontramos relações negativas entre a disciplina, (Retro-FUS), valência e rapidez (FTPS); entre afeto Pai e 
afeto Mãe e rapidez; entre refeições e rapidez. Assim como relações negativas entre as dimensões finanças, refeições (Retro-FUS) e conectividade (FTPS).

\section{Discussão}

O objectivo inicial deste estudo era traduzir a validar para português a Escala Retrospectiva de Imprevisibilidade Familiar, de Ross e McDuff (2008); adicionalmente, estudou-se as relações entre a imprevisibilidade familiar retrospectiva e a perspectiva temporal de futuro em jovens adultos portugueses. O conceito de imprevisibilidade familiar tem-se revelado significativo para o desenvolvimento das crianças na família, sendo os estudos unânimes em considerar a imprevisibilidade familiar como factor de risco para o desenvolvimento das mesmas. Esta imprevisibilidade é traduzida na impossibilidade (ou falta de vontade) dos cuidadores em preservar as rotinas familiares que garantem a estabilidade comportamental, e a segurança nas expectativas relacionais entre os membros da família, particularmente com as crianças.

A imprevisibilidade familiar pode ser induzida por diversos factores, como a pobreza (Ross, 2006), situações clínicas dos cuidadores como alcoolismo, depressão, doenças crónicas que implicam internamentos e tratamentos que quebram rotinas familiares (Hill, Young, \& Nord, 1994; Ross, \& Hill, 2002; 2004). A instabilidade laboral e económica mais alargada (da qual não temos controlo) reflecte-se, de acordo com estudos revistos (Larson, Wilson, \& Beley, 1994; Voydanoff, 1990; 2005), no clima familiar, afectando particularmente as famílias já de si mais carenciadas, instalando-se facilmente um ciclo vicioso do qual é difícil sair sem ajuda. Os estudos revistos sugerem possibilidades de intervenção e prevenção ao explicitarem relações entre dimensões de rotinas familiares e maior controlo de expectativas de futuro (i.e., de planeamento e de expectativas de acções a realizar). Se algumas dimensões das dinâmicas familiares fogem mais ao controlo interno dos sujeitos (como o rendimento da família), outras, como o afecto, a disciplina parental as refeições, poderão ser um ponto de referência e estabilidade para o desenvolvimento das crianças. Os fundamentos teóricos subjacentes à noção de (im)previsibilidade familiar mostram como a previsibilidade promove a segurança interna e a possibilidade de planear o futuro ao longo do desenvolvimento.

\section{Referências}

Alarcão, M. \& Gaspar, F. M. (2007). Imprevisibilidade familiar e suas implicações no desenvolvimento individual e familiar. Paidéia, 17(36), 89-102.

Bandura, A. (2001). Social cognitive theory: An agentic perspective. Annual Review of Psychology, 52, 1-26. doi: 10.1146annurev.psych.52.1.1

Bowlby, J. (1988). A secure base. Clinical applications of attachment theory. London: Routhedge.

Bretherton, I., \& Munholland, K. A. (2008). Internal working models in attachment relationships: Elaborating a central construct in attachment theory. In
J. Cassidy, \& P. R. Shaver (Eds.), Handbook of attachment: Theory, research, and clinical applications ( $2^{\text {nd }}$ ed.), pp.102-127, New York: Guilford Press.

Cohen, J. (1988). Statistic power analysis for the behavioral sciences, 2th ed., Hillsdale, N.J.: Laurence Erlbaum Associates.

Hill, E. M., Jenkins, J., \& Farmer, L. (2008). Family unpredictability, future discounting, and risk taking. The Journal of Socio-Economics, 37, 1381-1396. doi: 10.1016/j.socec.2006.12.081

Hill, E. M., Young, J. P., \& Nord, J. L. (1994). Childhood adversity, attachment security, and adult relationships: A preliminary study. Ethology and Sociobiology, 15(5-6), 323-338.

10.1016/0162/-3095(94)90006-X

Howar-Rodrigues, A. B. C., Andrade, A. L., \& Tokumaru, R. S. (2012). Construção e validação da Escala de Imprevisibilidade Familiar na Infância (EIFI). Psicologia, Reflexão e Crítica, 25(2), 221-220.

Howar-Rodrigues, A. B. C., Tokumaru, R. S. (2014). Scale of family unpredictability during childhood: Validity evidence. Paidéia, 24(57), 11-20. doi: 10.1590/1982-43272457201403

Hunziker, M. H. L. (2005). O desamparo aprendido revisitado: estudos com animais. Psicologia: Teoria e Pesquisa, 21(2), 131-139.

Hunziker, M. H. L., \& dos Santos, C. V. (2007). Learned helplessness: effects of response requirement and interval between treatment and testing. Behavioural Processes, 76, 183-191. doi: 10.1016/j.beproc.2007.02.012

Husman, J., \& Shell, D. F. (2008). Beliefs and perceptions about the future: A measurement of future time perspective. Learning and Individual Differences, 18, 166-175. doi: 10.1016/j.lindif.2007.08.001

Kilday, Z. (2013). Inescapable aversive stimulus decreases subsequent escape responding in humans: An investigation of the learned helplessness effect in a 3D virtual environment. Electronic Theses \& Dissertations. Paper 834.

Laghi, F., D’Alessio, M., Pallini, S., \& Baiocco, R. (2009). Attachment representations and time perspective in adolescence. Social Indicators Research, 90(2), 181-194. doi: 10.1007/s11205-008-9249-0

Larson, J. H., Wilson, S. M., \& Beley, R. (1994). The impact of job insecurity on marital and family relationships. Family Relations, 43(2), 138-143.

Lens, W., Paixão, M. P., Herrera, D., \& Grobler, A. (2012). Future time perspective as a motivational variable: Content and extension of future goals affect the quantity and quality of motivation. Japanese Psychological Research, 54(3), 321-333. doi:10.1111/j.1468-5884.2012.00520.x

LoLordo, V. M., \& Overmier, J. B. (2011). Trauma, learned helplessness, its neuroscience, and implications for PTSD. In T. R. Schachtman \& S. Reilley (Eds.), Associative Learning and Conditioning Theory: Human and non-human applications (ch.6, pp, 121-167). Oxford: Oxford University Press. 
Machado, T. S. (2004). Vinculação e comportamentos anti-sociais. In A. C. Fonseca (Ed.). Comportamento anti-social e crime. Da infância à idade adulta (pp.291-321). Coimbra: Almedina.

Machado. T. S. (2007). Padrões de vinculação aos pais em adolescentes e jovens adultos e adaptação à Universidade. Revista Portuguesa de Pedagogia, 41(2), 5-28.

Machado, T. S. (2009). Vinculação aos pais: retorno às origens. Psicologia, Educação e Cultura, XIII(1), 139-156.

Machado, T. S., \& Correia, I. (2013). Imprevisibilidade familiar e suporte social percebido por famílias em intervenção precoce e famílias de comunidade. In A. Pereira, M. Calheiros, et al. (Org.). Livro de Atas VIII Simpósio Nacional de Investigação em Psicologia (pp.257-264). APP.

Nenty, H. J., \& Edna, O. N. (2009). Influence of gender and learned helplessness on some mathematics-related cognitive behavior of Lesotho of senior secondary school students. Gender \& Behaviour, 7(1), 2125-2139.

Nodar, M. (2012). Chaotic environments and adult children of alcoholics. The Professional Counselor: Research and Practice, 2(1), 43-47.

O'Dweyer, C. (2000). Imagining one's future: A projective approach to Christian maturity. Roma: Editrice Pontificia Università Gregoriana.

Oliveira, E. C., \& Hunziker, M. H. (2014). Longitudinal investigation on learned helplessness tested under negative and positive reinforcement involving stimulus control. Behavioural Processes, 106, 160-167. doi: 10.1016/j.beproc.2014.03.009.

Paixão, M. P. (2004). A dimensão temporal do futuro na elaboração de objectivos pessoais e organização de projectos vocacionais. Psychologica, (Extra-série), 273-286.

Peterson, C., Maier, S. F., \& Seligman, M. E. P. (1993). Learned helplessness. A theory for the age of personal control. New York: Oxford University Press.

Ross, L. T., \& Hill, E. M. (2000). The Family Unpredictability Scale: Reliability and validity. Journal of Marriage and Family, 62(2), 549-562. doi:10.1007/s10826-007-9138-1

Ross, L. T., \& Hill, E. M. (2002). Childhood unpredictability, schemas for unpredictability, and risk taking. Social Behavior and Personality, 30(5), 453-474.

Ross, L. T., \& Hill, E. M. (2004). Comparing alcoholic and nonalcoholic parents on the family unpredictability scale. Psychological Reports, 94(3), 1385-1391. doi: 10.2466/pr0.943c.

Ross, L. T., \& Hill, E. M., \& Low, B. S. (1997). The role of future unpredictability in human risk-taking. Human Nature, 8(4), 287-325.

Ross, L. T., \& McDuff, J. A. (2008). The retrospective family unpredictability Scale: Reliability and validity. Journal of Child and Family Studies, 17(1), 13-27. doi: 10.1007/s10826-007-9138-1

Ross, L. T., \& Wynne, S. (2010). Parental depression and divorce and adult children's well-being: The role of family unpredictability. Journal of Family Studies, 19, 757-761. doi: 10.1007/s10826-010-9366-7

Seligman, M. E. P., \& Maier, S. F. (1967). Failure to escape traumatic shock. Journal of Experimental Psychology, 74(1), 1-9.

Sorrenti, L., Fillipello, P., Costa, S., \& Buzzai, C. (2014). Preliminari evaluation of a self-report tool for learned helplessness and mastery orientation in Italian students. Mediterranean Journal of Clinical Psychology, II(3), doi: 10.6092/2282-1619/2014.2.1024

Trad, P. V. (1986). Infant depression. Paradigms and paradoxes. New York: Springer-Verlag.

Voydanoff, P. (1990). Economic distress and family relations: A review of the eighties. Journal of Marriage and the Family, 52, 1099-1115. doi: 10.2307/353320

Voydanoff, P. (2005). Toward a conceptualization of perceived work-family fit balance: A demands and resources approach. Journal of Marriage and Family, 67(4), 822-836. doi: 10.1111/j.1741-3737.2005.00178.x

Zimbardo, P. G., \& Boyd, J. N. (1999). Putting time in perspective: A valid, reliable individual-differences metric. Journal of Personality and Social Psychology, 77(6), 1271-1288. 